\title{
Light Hadron Physics at BESIII
}

\author{
Shuang-shi Fang ${ }^{* \dagger}$ \\ Institue of High Energy Physics, CAS, Beijing, China \\ E-mail: Eangssdihep.ac.cn
}

With a sample of $1.3 \times 10^{9} \mathrm{~J} / \psi$ and $4.5 \times 10^{8} \psi(3686)$ events collected with the BESIII detector, the recent results on the light hadron physics, including study of the light meson spectroscopy, search for the strangeonium-like structure and the light meson decay dynamics, are presented.

The 9th International Workshop on Chiral Dynamics,

September 17-20, 2018

Durham, 2018

\footnotetext{
*Speaker.

${ }^{\dagger}$ On behalf of the BESIII Collaboration
} 


\section{Introduction}

At present our theoretical understanding of the light hadron spectrum remains based largely on the quark model due to the failure of the application of the perturbative theory in the low energy regime. In addition to the coventional hadrons predicted by the quark model, however, one of the clearest predictions of Quantum chromodynamics (QCD) is the presence of exotic hadrons, e.g., glueballs, multiquark states and hybrids. The search for these exotic states has been taken for many years and many candidates have been reported by different experiments, but none of them were unambiguously identified to date.

The world's largest samples of $J / \psi$ and $\psi(3686)$ events produced from the $e^{+} e^{0}$ annihilations were collected at the BESIII detector, which offer an opportunity to study the light hadron spectroscopy and to investigate the light hadron decays. Based on the samples of $1.3 \times 10^{9} \mathrm{~J} / \psi$ events and $1.06 \times 10^{8} \psi(3686)$ events, in this talk we present the progresses on the light meson spectroscopy and light meson decays.

\section{Light meson spectroscopy}

\subsection{The structures around $1.84 \mathrm{GeV} / \mathrm{c}^{2}$}

In 2005 the $\mathrm{X}(1835)$ resonance was observed in $J / \psi \rightarrow \gamma \pi^{+} \pi^{-} \eta^{\prime}[\mathrm{W}]$, which then stimulated theorectial speculstions on its nature. At BESIII, this structure was confirmed in the same decay channel [[] and other similar structures around $1.85 \mathrm{GeV} / \mathrm{c}^{2}$ were observed. To understand their nature, further study is strongly needed, in particular, in searching for new decay modes.

In this talk we present a study of $J / \psi \rightarrow \gamma K_{S} K_{S} \eta$ [目] decays using a sample of (1310.6 \pm $10.5) \times 10^{6} \mathrm{~J} / \psi$ events collected with the BESIII detector. By requiring that $M_{K_{S} K_{S}}$ is in the $f_{0}(980)$ mass region, $M_{K_{S} K_{S}}<1.1 \mathrm{GeV} / \mathrm{c}^{2}$, the structure around $1.85 \mathrm{GeV} / \mathrm{c}^{2}$ shown in Fig. W (a) is clearly seen. A partial wave anlaysis (PWA) is performed and the spin parity of the $X(1835)$ is determined to be $J^{P C}=0^{-+}$. The mass and width are measured to be $1844 \pm 9(\text { stat })_{-25}^{+16}($ syst $) \mathrm{MeV} / c^{2}$ and $192_{-17}^{+20}(\text { stat })_{-43}^{+62}($ syst $) \mathrm{MeV}$, respectively, which are consistent with the results obtained by BESIII in $J / \psi \rightarrow \gamma \pi^{+} \pi^{-} \eta^{\prime}[$ [ $]$ ]. Figure W (b) shows the comparison to the BESIII results of the masses and widths of the $X(p \bar{p}), X(1835), X(1870) X(1840)$ and $X(1810)$. The mass of $X(1840)$ is in agreement with $X(p \bar{p})$, while its width is significantly broader. Therefore, based on these data, one cannot determine whether $X(1840)$ is a new state or the signal of a $3\left(\pi^{+} \pi^{-}\right)$decay mode of $X(p \bar{p})$. Further study, including an amplitude analysis to determine the spin and parity of the $X(1840)$, is needed to establish the relationship between these experimental observations.

Most recently we performed a study on the decay $J / \psi \rightarrow \gamma \gamma \phi[$ [ $]$. Two structures around 1475 $\mathrm{MeV} / \mathrm{c}^{2}$ and $1835 \mathrm{MeV} / \mathrm{c}^{2}$ are observed in the $\gamma \phi$ invariant mass spectrum for the first time. With a fit on the $\gamma \phi$ invariant mass, which takes into account the interference between the two structures, and a simple analysis of the angular distribution, the structure around $1475 \mathrm{MeV} / \mathrm{c}^{2}$ is found to favor an assignment as the $\eta(1475)$ and the mass and width for the structure around $1835 \mathrm{MeV} / \mathrm{c}^{2}$ are consistent with the $X(1835)$. The statistical significances of the two structures are $13.5 \sigma$ and $6.3 \sigma$, respectively. A fit on the polar angle distribution of the radiative photon favor $J^{P C}=0^{-+}$ assignment for the two resonances. The obtained resonant parameters and $J^{P C}$ supports the two new observed resonances are $\eta(1475)$ and $X(1835)$, respectively, and this is for the first time we 

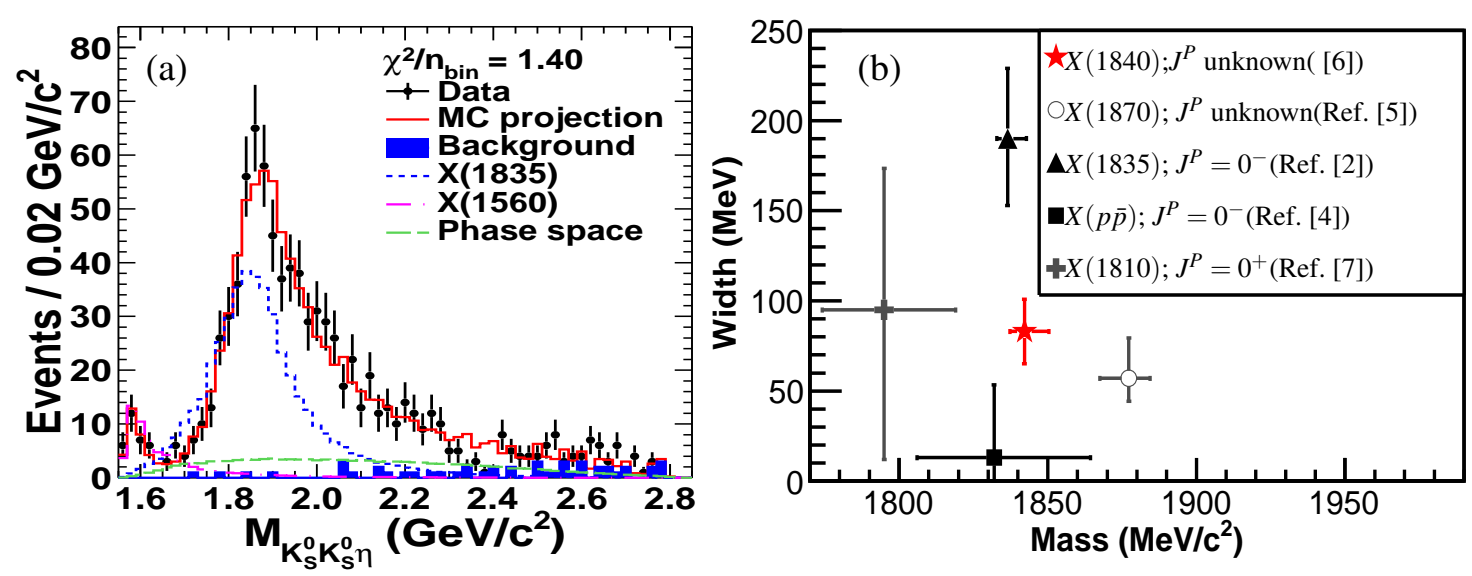

Figure 1: (a) $K_{S}^{0} K_{S}^{0} \eta$ invariant mass spectrum for events with the requirement $M_{K_{S}^{0} K_{S}^{0}}<1.1 \mathrm{GeV} / c^{2}$ and PWA projections; (b) comparisons of observations at BESIII. The error bars include statistical, systematic, and, where applicable, model uncertainties.

observed $\eta(1475)$ and $X(1835)$ decaying into $\gamma \phi$ final states, which indicate that both $\eta(1475)$ and $X(1835)$ contain a sizeable $s \bar{s}$ component.
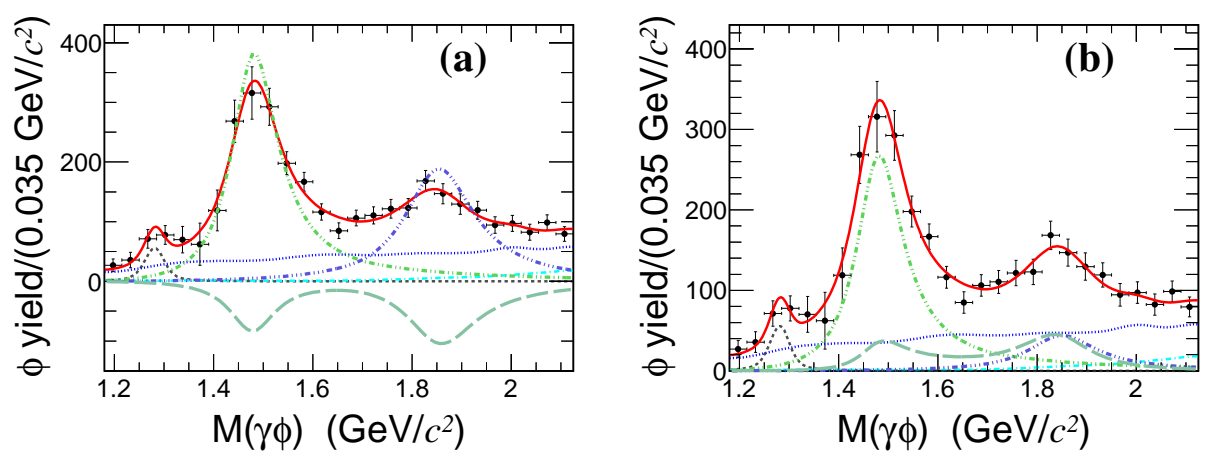

Figure 2: Fits to the $M_{\gamma \phi}$ distributions (two combinations per event) for the case of (a) constructive and (b) destructive interference. The dots with error bars are the data. The (red) solid, (green) dash double-dotted, dash triple-dotted, (black) dashed, (blue) dotted and long-dashed lines are the fit results, the structures around $1.47,1.83 \mathrm{GeV} / c^{2}, f_{1}(1285)$, backgrounds and interference contributions, respectively.

\subsection{PWA of $J / \psi \rightarrow \gamma K_{S} K_{S}$ [可]}

An amplitude analysis of the $K_{S} K_{S}$ system produced in radiative $J / \psi$ decays is performed with both mass-dependent and mass-independent approaches. The mass-dependent analysis is performed by parameterizing the $K_{S} K_{S}$ invariant mass spectrum as a sum of Breit-Wigner line shapes. Additionally, a mass-independent analysis is performed to extract a piecewise function that describes the dynamics of the $K_{S} K_{S}$ system while making minimal assumptions about the properties and number of poles in the amplitude. The dominant amplitudes in the mass-dependent analysis include the $f_{0}(1710), f_{0}(2200)$, and $f_{2}^{\prime}(1525)$. The dominant scalar amplitudes come from the 
$f_{0}(1710)$ and $f_{0}(2200)$, which have production rates in radiative $J / \psi$ decays consistent with predictions from lattice QCD for a $0^{+}$glueball. The production rate of the $f_{0}(1710)$ is about one order of magnitude larger than that of the $f_{0}(1500)$, which suggests that the $f_{0}(1710)$ has a larger overlap with the glueball state compared to the $f_{0}(1500)$. The tensor spectrum is dominated by the $f_{2}^{\prime}(1525)$ and $f_{2}(2340)$. Recent Lattice QCD predictions for the production rate of the pure gauge tensor glueball in radiative $J / \psi$ decays are consistent with the large production rate of the $f_{2}(2340)$ in the $K_{S} K_{S}, \eta \eta[\square]$, and $\phi \phi$ [Ш]] spectra. The mass-dependent results are consistent with the results of a mass-independent amplitude analysis of the $K_{S} K_{S}$ invariant mass spectrum. The mass-independent results are useful for a systematic study of hadronic interactions. A more comprehensive study of the light scalar meson spectrum should benefit from the inclusion of these results with those of similar reactions.
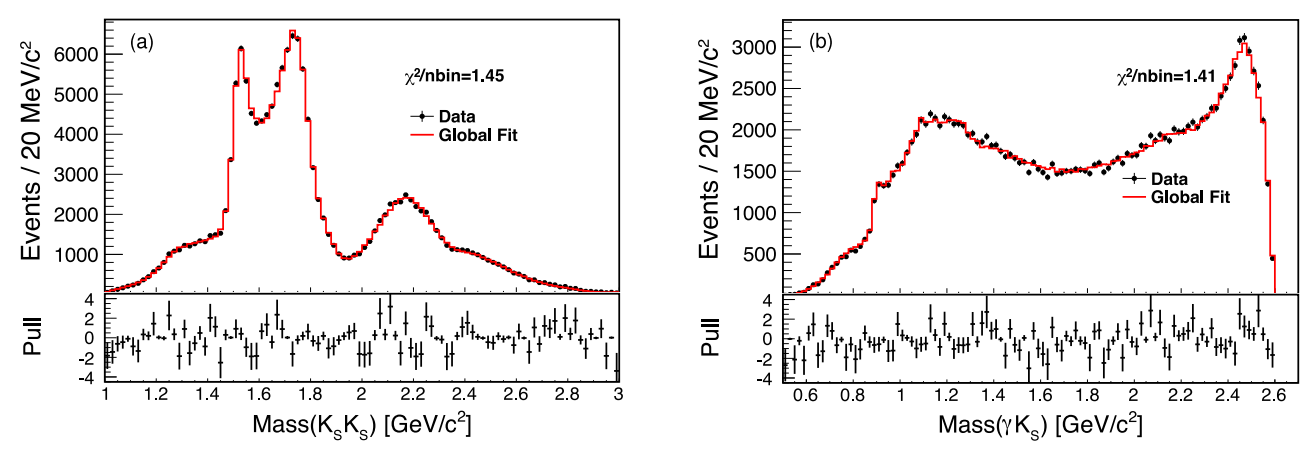

Figure 3: (Color online) Distributions of the (a) $K_{S} K_{S}$ and (b) $\gamma K_{S}$ invariant mass spectra. Markers with error bars are the data and the red histograms are the fit results for the MD analysis. The pull distributions ((data-fit)/error) are shown below each plot.

\subsection{Search for the strangenonium-like structure $Z_{s}$ in $e^{+} e^{-} \rightarrow \phi \pi \pi$ [12]]}

A charged charmoniumlike structure, $Z_{c}(3900)$, was observed in the $\pi^{ \pm} J / \psi$ final states by the BESIII and Belle experiments [[131, [4]], which inspired extensive discussions of their nature, and the reasonable interprestations are tetraquark states, molecular or hadroquarkonium states due to these structures carrying charge and prominently decaying into a pion and a conventional charmonium state. By replacing the $c \bar{c}$ pair in the $Z_{c}$ structure with an $s \bar{s}$, it is possible to consider an analogous $Z_{s}$ structure. Similar to $Y(4260) \rightarrow J / \psi \pi^{+} \pi^{-}$in which the $Z_{c}(3900)$ was observed [153, [4]], the process $\phi(2170) \rightarrow \phi \pi^{+} \pi^{-}$is considered as a unique place to search for the $Z_{s}$ structure, as the $\phi(2170)$ is regarded as the strangeoniumlike states analogy to $Y(4260)$ in charmonium sector.

Using a data sample corresponding to an integrated luminosity of $(108.49 \pm 0.75) \mathrm{pb}^{-1}$, taken at a center-of-mass energy of $2.125 \mathrm{GeV}$ with the BESIII detector, we present a search for the $Z_{s}$ structure in the process $e^{+} e^{-} \rightarrow \phi \pi \pi$. No $Z_{s}$ signal is observed in the $\phi \pi$ invariant mass spectrum, and corresponding upper limits on the cross sections of $Z_{s}$ production at the $90 \%$ C.L. are determined for different mass and width hypotheses, as displayed in Fig. 6 . The results around 1.4 $\mathrm{GeV} / c^{2}$ indicate the ISPE mechanism at $K^{*} \bar{K}$ threshold is not as significant as predicted in Ref. [ए5]]. Further study with larger statistics is essential to examine the existence of the $Z_{s}$ structure and test the ISPE mechanism. 

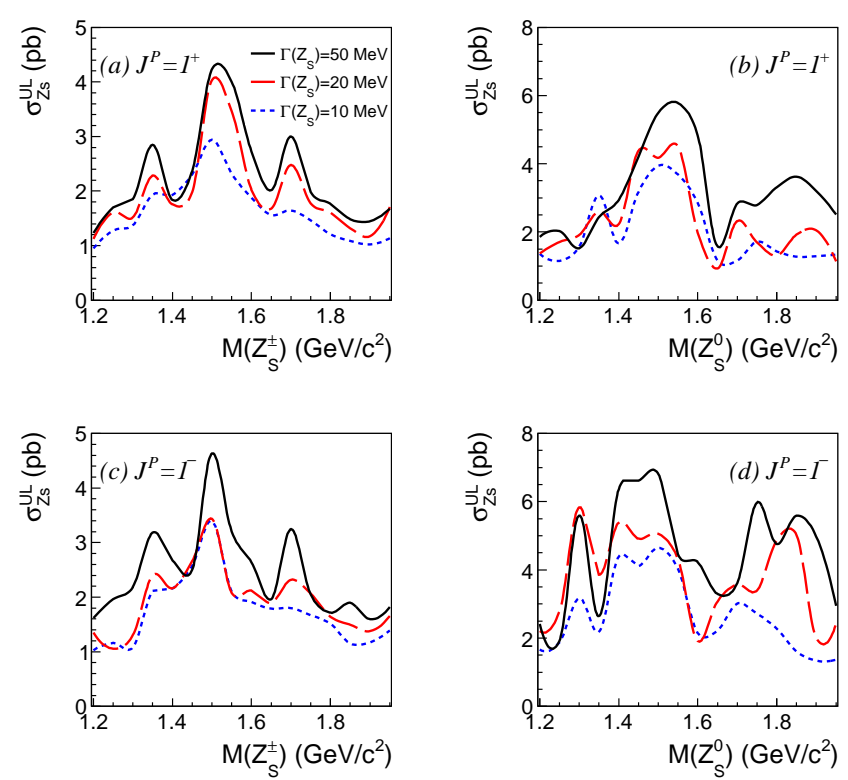

Figure 4: The upper limits at $90 \%$ C.L. on the differential cross sections of $Z_{s}$ as a function of assumed signal peak mass for the cases (a) $J^{P}=1^{+}$of $Z_{s}^{ \pm}$, (b) $J^{P}=1^{+}$of $Z_{s}^{0}$, (c) $J^{P}=1^{-}$of $Z_{s}^{ \pm}$, and (d) $J^{P}=1^{-}$of $Z_{s}^{0}$. The dotted, dashed and solid lines are the results of $\Gamma=10,20$, and $50 \mathrm{MeV}$, respectively.

\section{Light meson decays}

3.1 $\eta^{\prime} \rightarrow \pi^{+} \pi^{-} \eta$ [10] and $\eta^{\prime} \rightarrow \pi^{+(0)} \pi^{-(0)} \eta$ [117]
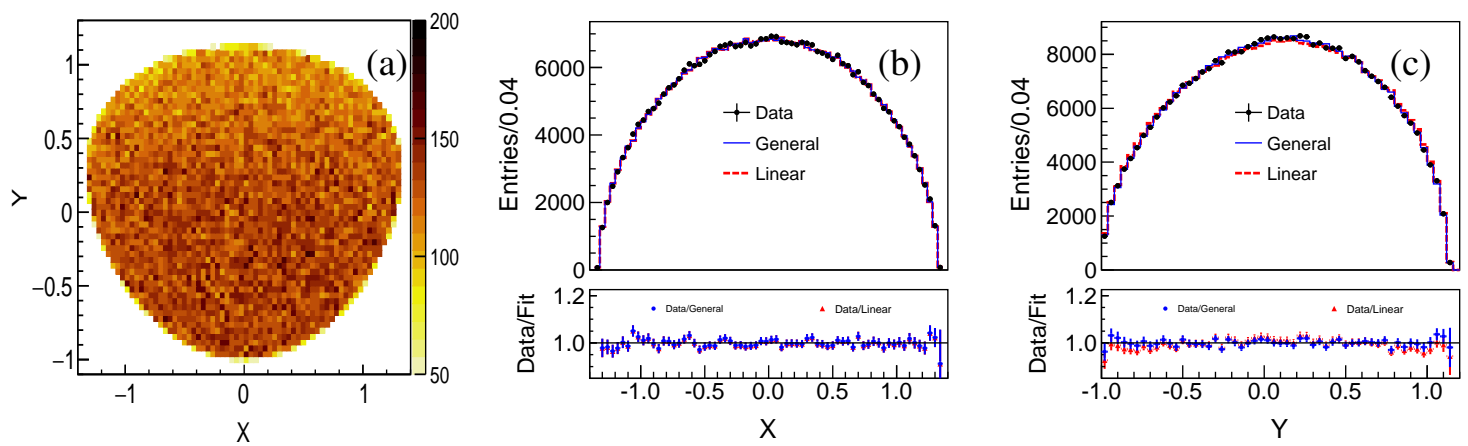

Figure 5: (a) The experimental Dalitz plot for the decay $\eta^{\prime} \rightarrow \pi^{+} \pi^{-} \eta$ in terms of the variables $X$ and $Y$ with the $\pi^{+} \pi^{-} \eta$ mass in the $\eta^{\prime}$ mass region. The corresponding projections on variables $X$ and $Y$ are shown in (b) and (c), respectively, where the dashed histograms are from MC of $\eta^{\prime} \rightarrow \pi^{+} \pi^{-} \eta$ events generated with phase space. The solid histograms are the fit results described in the text.

Initial BESIII $\eta^{\prime} \rightarrow \pi^{+} \pi^{-} \eta$ Dalitz plot analysis [ए]] was based on 2009 data and both the general and linear representations were used. The most recent BESIII analysis [ए]] uses nearly background free samples of $3.5 \times 10^{5} \eta^{\prime} \rightarrow \eta \pi^{+} \pi^{-}$events and $5.6 \times 10^{4} \eta^{\prime} \rightarrow \eta \pi^{0} \pi^{0}$ events 
from $1.31 \times 10^{9} \mathrm{~J} / \psi$. The goal was determination of the Dalitz plot parameters for the two decay modes and a search for the cusp at the $\pi^{0} \pi^{0} \rightarrow \pi^{+} \pi^{-}$threshold in $\eta^{\prime} \rightarrow \eta \pi^{0} \pi^{0}$. For the decay of $\eta^{\prime} \rightarrow \eta \pi^{+} \pi^{-}$, the fit results for the two representations, which are shown in Fig. [1].

For the $\eta^{\prime} \rightarrow \eta \pi^{+} \pi^{-}$decay, the results, superseding the previous BESIII measurement [ए]], are not consistent with the measurement from VES and the theoretical predictions within the framework of $U(3)$ chiral effective field theory in combination with a relativistic coupled-channels method (Chiral Unitary Approach - ChUA) [18]]. In particular for the cofficient $a$, the discrepancies are about four standard deviations. On the other hand large- $N_{C}$ ChPT prediction at next-to-next-toleading order [ए耳] is consistent with the measured $a$ value due to the large theoretical uncertainty. For the cofficient $c$ violating charge conjugation, the fitted values are consistent with zero within one standard deviation for both representations.

In case of $\eta^{\prime} \rightarrow \eta \pi^{0} \pi^{0}$, the results are in general consistent with the previous measurements and theoretical predictions within the uncertainties from both sides. The latest results [20] reported by the A2 experiment are also in agreement with those obtained from BESIII. We notice a discrepancy of 2.6 standard deviations for parameter $a$ between $\eta^{\prime} \rightarrow \eta \pi^{+} \pi^{-}$and $\eta^{\prime} \rightarrow \eta \pi^{0} \pi^{0}$ modes. The present results are not precise enough to firmly establish isospin violation and additional effects, e.g., radiative corrections [2]] and $\pi^{+} / \pi^{0}$ mass difference should be considered in the future experimental and theoretical studies.

\subsection{Amplitude analysis of $\eta^{\prime} \rightarrow \pi^{+(0)} \pi^{-(0)} \pi^{0}$ [22]}

At first, the low intensity process $\eta^{\prime} \rightarrow \pi^{+} \pi^{-} \pi^{0}$ may be considered to come from $\pi^{0}-\eta$ mixing in the dominating decay $\eta^{\prime} \rightarrow \pi^{+} \pi^{-} \eta$ [23]. This would offer a possibility to determine precisely $u-d$ quark mass difference from the branching fraction ratio of the two processes. However, a recent analysis shows that even at tree level other terms are needed [24]. In addition the decay amplitudes are strongly affected by the intermediate resonances. Therefore the mixing of $\pi^{0}-\eta$ and $u-d$ quark mass difference cannot be extracted in a simple way.

The common amplitude analysis of the decays $\eta^{\prime} \rightarrow \pi^{+} \pi^{-} \pi^{0}$ and $\eta^{\prime} \rightarrow \pi^{0} \pi^{0} \pi^{0}$ is performed using isobar model. The fit results illustrated by the invariant mass spectra of $\pi^{+} \pi^{-}, \pi^{+} \pi^{0}$ and $\pi^{-} \pi^{0}$ (Fig. (6) show significant $P$-wave contribution from $\eta^{\prime} \rightarrow \rho^{ \pm} \pi^{\mp}$ in $\eta^{\prime} \rightarrow \pi^{+} \pi^{-} \pi^{0}$. The branching fraction $\mathscr{B}\left(\eta^{\prime} \rightarrow \rho^{ \pm} \pi^{\mp}\right)$ is determined to be $\left(7.44 \pm 0.60 \pm 1.26 \pm 1.84_{\text {model }}\right) \times 10^{-4}$. In addition to the non-resonant $S$-wave, the resonant $\pi-\pi S$-wave with a pole at $(512 \pm 15)-i(188 \pm$ 12) $\mathrm{MeV}$, interpreted as the broad $\sigma$ meson, plays essential role in the $\eta^{\prime} \rightarrow \pi \pi \pi$ decays. Due to the large interference between non-resonant and resonant $S$-waves, only the sum is used to describe the $S$-wave contribution, and the branching fraction is determined to be $\mathscr{B}\left(\eta^{\prime} \rightarrow \pi^{+} \pi^{-} \pi^{0}\right)_{S}=$ $\left(37.63 \pm 0.77 \pm 2.22 \pm 4.48_{\text {model }}\right) \times 10^{-4}$.

For $\eta^{\prime} \rightarrow \pi^{0} \pi^{0} \pi^{0}$, the $P$-wave contribution in two-body rescattering is forbidden by Bose symmetry. The corresponding branching fraction is measured to be $\mathscr{B}\left(\eta^{\prime} \rightarrow \pi^{0} \pi^{0} \pi^{0}\right)=(35.22 \pm$ $0.82 \pm 2.60) \times 10^{-4}$, which is about two times larger than GAMS measurement of $(16 \pm 3.2) \times$ $10^{-4}[$ [25].

\subsection{Observation of $\eta^{\prime} \rightarrow \gamma e^{+} e^{-}$[26]}

Electromagnetic (EM) Dalitz decays of light pseudoscalar mesons, $P \rightarrow \gamma l^{+} l^{-}\left(P=\pi^{0}, \eta, \eta^{\prime}\right.$; $l=e, \mu)$, play an important role in revealing the structure of hadrons and the interaction mechanism 

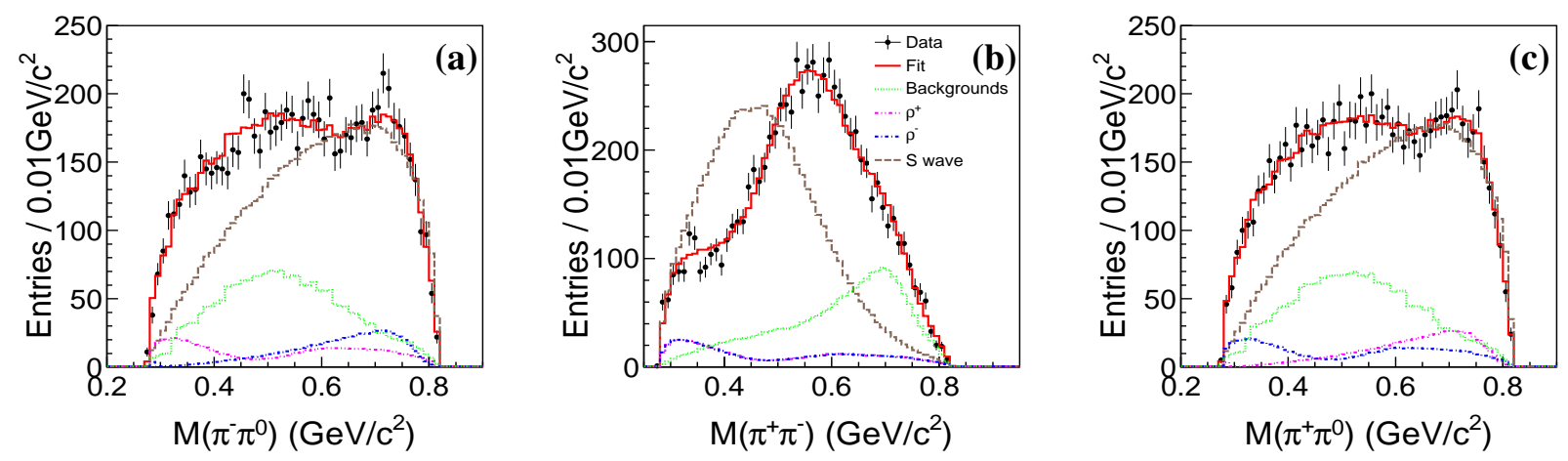

Figure 6: Comparison of the invariant mass distributions of (a) $\pi^{-} \pi^{0}$, (b) $\pi^{+} \pi^{-}$, (c) $\pi^{+} \pi^{0}$ (dots with error bars) and the fit projections (solid histograms). The dotted, dashed, dash-dotted, and dash-dot-dotted histograms show the contributions from background, $S$ wave, $\rho^{-}$, and $\rho^{+}$, respectively.

between photons and hadrons [DZ]. In this work, we report the first observation of the Dalitz decay $\eta^{\prime} \rightarrow \gamma e^{+} e^{-}$and the branching fraction $\mathscr{B}\left(\eta^{\prime} \rightarrow \gamma e^{+} e^{-}\right)$is measured to be $(4.69 \pm 0.20 \pm 0.23) \times$ $10^{-4}$.
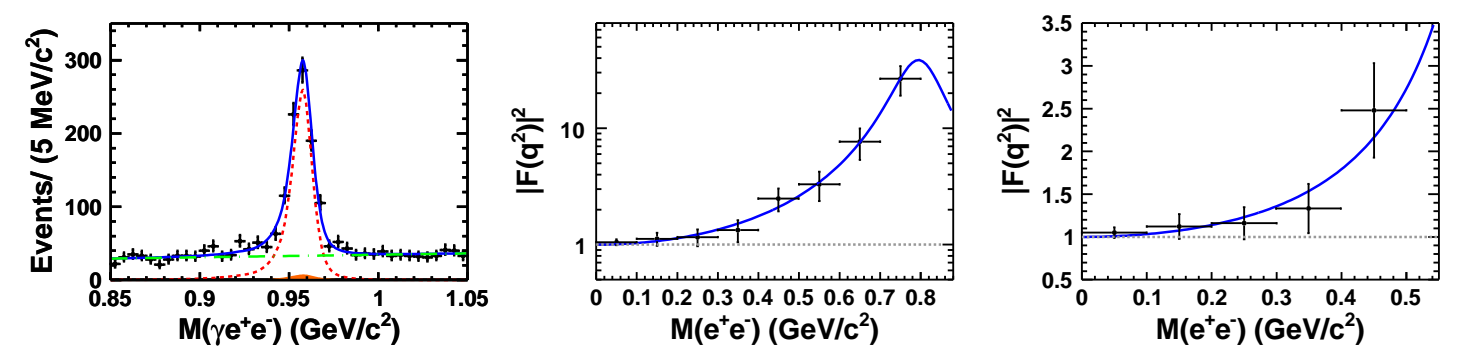

Figure 7: Invariant $\gamma e^{+} e^{-}$mass distribution for the selected signal events. The (black) crosses are the data, the (red) dashed line represents the signal, the (green) dot-dashed curve shows the non-peaking background shapes, the (orange) shaded component is the shape of the $J / \psi \rightarrow \gamma \eta^{\prime}, \eta^{\prime} \rightarrow \gamma \gamma$ peaking background events. The total fit result is shown as the (blue) solid line.

In addition, we present measurements of the transition form factor (TFF) as a function of $M\left(e^{+} e^{-}\right)$. Our TFF results can be described with a single pole parameterization of $F\left(q^{2}\right)=$ $\frac{1}{\left(1-q^{2} / \Lambda^{2}\right)}$, with mass and width parameters of $\Lambda_{\eta^{\prime}}=(0.79 \pm 0.04 \pm 0.02) \mathrm{GeV}$, and $\gamma_{\eta^{\prime}}=(0.13 \pm$ $0.06 \pm 0.03) \mathrm{GeV}$, respectively. The slope of the TFF corresponds to $(1.60 \pm 0.17 \pm 0.08) \mathrm{GeV}^{-2}$ and agrees within errors with the VMD model predictions. The uncertainty of the $\eta^{\prime}$ transition form factor slope matches the best determination in the space-like region from the CELLO collaboration $b_{\eta^{\prime}}=(1.60 \pm 0.16) \mathrm{GeV}^{-2}$ [?], and improves the previous determination of the slope in the time-like region $b_{\eta^{\prime}}=(1.7 \pm 0.4) \mathrm{GeV}^{-2}$ [27].

\subsection{Precision study of $\eta^{\prime} \rightarrow \gamma \pi^{+} \pi^{-}$decay dynamics [28]}

The anomalous process $\eta^{\prime} \rightarrow \gamma \pi^{+} \pi^{-}$is the second most probable decay of the $\eta^{\prime}$ meson and frequently used for $\eta^{\prime}$ tagging. In the VMD model the main contribution to the decay comes from $\eta^{\prime} \rightarrow \gamma \rho^{0}\left[\right.$ [29]. For BESIII analysis a low background data sample of $9.7 \times 10^{5} \eta^{\prime} \rightarrow \gamma \pi^{+} \pi^{-}$ 
decays candidates is selected. The distribution of the $\pi^{+} \pi^{-}$invariant mass, $M\left(\pi^{+} \pi^{-}\right)$, is displayed in Fig. 8. The $\rho^{0}-\omega$ interference is seen for first time in this decay. In the model-dependent approach the data can not be described with Gounaris-Sakurai parameterisation [B]] of the $\rho^{0}$ and the $\omega$ contributions including the interference. The fit performance gets much better after including the box anomaly, Fig. $8($ a), with a statistical significance larger than $37 \sigma$. An alternative fit was performed by replacing the box anomaly with $\rho^{0}(1450)$, Fig. 8 (b), by fixing its mass the width to the world average values. The fit is slightly worse but it still provides a reasonable description of the data.

Using model-independent approach of Ref. [B] ] and including $\rho^{0}-\omega$ mixing the pion vector form factor $F_{V}(s)$ (where $s=M^{2}\left(\pi^{+} \pi^{-}\right)$) and amplitudes for $\eta / \eta^{\prime} \rightarrow \gamma \pi^{+} \pi^{-}$decays are proportional to $P(s) \cdot \Omega(s)$ where $P(s)$ is a reaction specific term, $P(s)=1+\kappa s+\lambda s^{2}+\xi \cdot B W_{\omega}+\mathscr{O}\left(s^{4}\right)$, $\Omega(s)$ is the Omnes function describing $\pi-\pi$ interactions with $L=1$. For $\eta \rightarrow \gamma \pi^{+} \pi^{-}$only the linear term $\kappa=1.32 \pm 0.13 \mathrm{GeV}^{-2}$ [32, [33] is needed. The fit to the BESIII $\eta^{\prime} \rightarrow \gamma \pi^{+} \pi^{-}$ data is shown in Fig. $\mathbb{8}\left(\mathrm{c}\right.$ (c), it yields $\kappa=0.992 \pm 0.039 \mathrm{GeV}^{-2}, \lambda=-0.523 \pm 0.039 \mathrm{GeV}^{-4}$, $\xi=0.199 \pm 0.006$ with the fit goodness $\chi^{2} / \mathrm{ndf}=145 / 109$. The presence of the quadratic term is consistent with recent calculations including intermediate $\pi^{ \pm} a_{2}^{\mp}$ state [B4]].
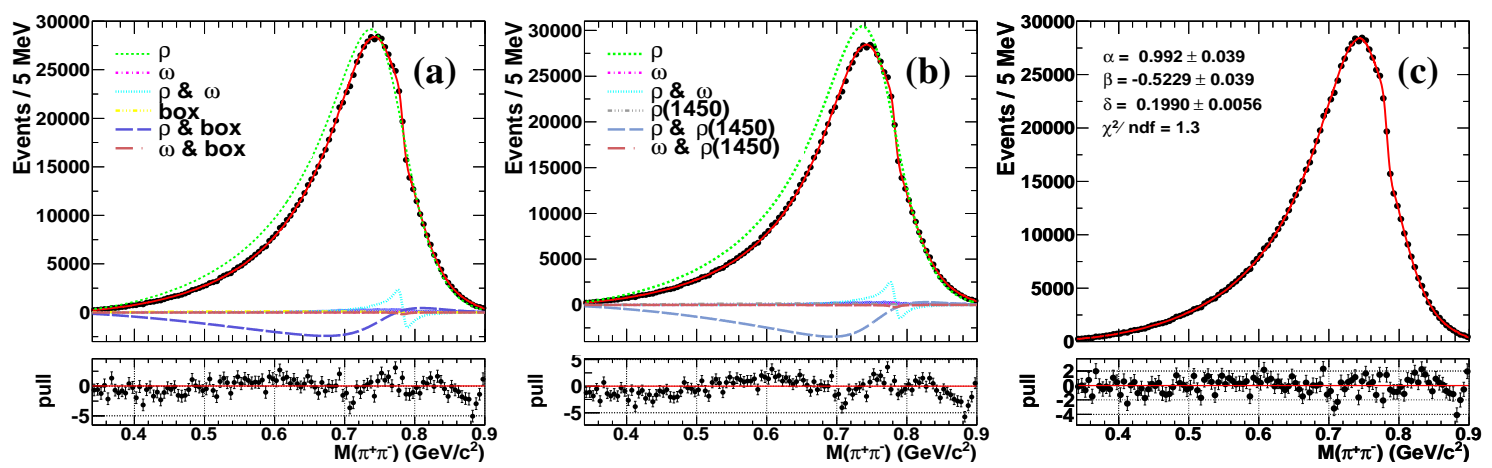

Figure 8: The results of the model-dependent fits to $M\left(\pi^{+} \pi^{-}\right)$with (a) $\rho^{0}-\omega-$ box anomaly and (b) $\rho^{0}-\omega-\rho^{0}(1450)$. (c) The results of model-independent fit with $\omega$ interference.

\subsection{Observation of $a_{0}(980)-f_{0}(980)$ mixing [35]}

The mixing mechanism in the system of $a_{0}(980)-f_{0}(980)$, which was first proposed in the late 1970s [36], is thought to be an essential approach to clarify the nature of these two mesons. Both $a_{0}(980)$ and $f_{0}(980)$ can decay into $K^{+} K^{-}$and $K^{0} \overline{K^{0}}$, which show a difference of $8 \mathrm{MeV} / c^{2}$ in the production mass threshold due to isospin breaking effects. The mixing amplitude between $a_{0}(980)$ and $f_{0}(980)$ is dominated by the unitary cuts of the intermediate two-kaon system and proportional to the phase-space difference between them. As a consequence, a narrow peak of about $8 \mathrm{MeV} / c^{2}$ in width is predicted between the charged and neutral $K \bar{K}$ mass thresholds, while the normal widths of $a_{0}(980)$ and $f_{0}(980)$ should be $50-100 \mathrm{MeV} / c^{2}$. The mixing mechanism has been studied extensively in various aspects, and many reactions have been discussed, but no quantitative experimental result has been firmly established yet.

Using data samples of $1.31 \times 10^{9} \mathrm{~J} / \psi$ events and $4.48 \times 10^{8} \psi(3686)$ events, we report the first observation of $a_{0}^{0}(980)-f_{0}(980)$ mixing in the decays of $J / \psi \rightarrow \phi f_{0}(980) \rightarrow \phi a_{0}^{0}(980) \rightarrow \phi \eta \pi^{0}$ 
and $\chi_{c 1} \rightarrow a_{0}^{0}(980) \pi^{0} \rightarrow f_{0}(980) \pi^{0} \rightarrow \pi^{+} \pi^{-} \pi^{0}$. Based on the input parameters of $f_{0}(980)$ and $a_{0}^{0}(980)$ in Refs. [37, [38], the signals of $f_{0}(980) \rightarrow a_{0}(980)$ and $a_{(980)} \rightarrow f_{0}(980)$ are observed for the first time with statistical significances of $7.4 \sigma$ and $5.5 \sigma$, as displyed in Fig. Q , respectively. The corresponding branching fractions of the mixing signal and the mixing intensities as well as the EM process of $J / \psi \rightarrow \phi a_{0}(980) \rightarrow \phi \eta \pi^{0}$ are also measured. Finally, the significance of the mixing signal is measured versus the values of the two coupling constants, $g_{a_{0} K^{+} K^{-}}$and $g_{f_{0} K^{+} K^{-}}$, and compared with theoretical predictions. In addition, the central values of the mixing intensities, $\xi_{f a}$ and $\xi_{a f}$, can be used to estimate the coupling constants of the $f_{0}(980)$ and $a_{0}^{0}(980)$ resonances. The results of this measurement help to deepen our understanding of the nature of the $f_{0}(980)$ and $a_{0}^{0}(980)$ mesons.

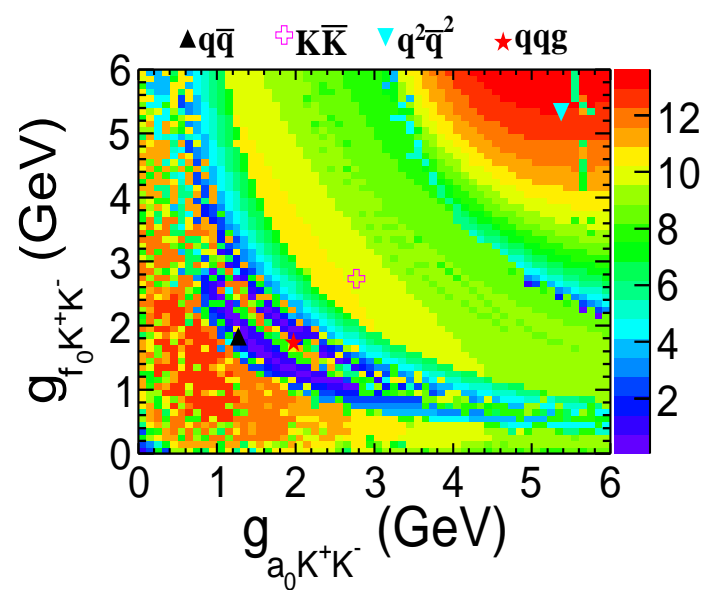

Figure 9: The statistical significance of the signal scanned in the two-dimensional space of $g_{a_{0} K^{+} K^{-}}$and $g_{f_{0} K^{+} K^{-}}$. The regions with higher statistical significance indicate larger probability for the emergence of the two coupling constants. The markers indicate predictions from various illustrative theoretical models.

\section{Summary and Outlook}

The decay of $J / \psi$ and $\psi(3686)$ is a rich field to be explored for the light hadron physics. With a sample of $1.3 \times 10^{9} \mathrm{~J} / \psi$ and $4.5 \times 10^{8} \psi(3686)$ events collected with the BESIII detector, the recent results on the light hadron physics, including study of the light meson spectroscopy, search for the strangeonium-like structure and the light meson decay dynamics, are presented.

During the 2018-2019 run, we took another sample of $8.7 \times 10^{9} \mathrm{~J} / \psi$ events. In total the 10 billion $J / \psi$ events, the world's largest sample produced from the $e^{+} e^{-}$annihilations, were accumulated at the BESIII experiment, which allow us to study the light hadron physics with unprecedent precision. In particular for the light meson decays, e.g. $\eta / \eta^{\prime}$ decays, this large data sample offer a unique opportunity to investigate their decay dynamics and search for the new physics through the rare or forbidden decays.

\section{References}

[1] M. Ablikim et al., Phys. Rev. Lett. 95, 262001 (2005). 
[2] M. Ablikim et al., Phys. Rev. Lett. 106, 072002 (2011).

[3] M. Ablikim et al., Phys. Rev. Lett. 115, 091803 (2015).

[4] M. Ablikim et al., Phys.Rev.Lett. 108,112003 (2012).

[5] M. Ablikim et al., Phys. Rev. Lett. 107, 182001 (2011).

[6] M. Ablikim et al., Phys. Rev. D 88, 091502 (2013).

[7] M. Ablikim et al., Phys.Rev. D 87, 032008 (2013).

[8] M. Ablikim et al., Phys. Rev. D 97, 051101(R) (2018).

[9] M. Ablikim et al., Phys. Rev. D 98, 072003 (2018).

[10] M. Ablikim et al., Phys. Rev. D 87, 092009 (2013).

[11] M. Ablikim et al., Phys. Rev. D 93, 112011 (2016).

[12] M. Ablikim et al., Phys. Rev. D 99, 011101(R) (2019).

[13] M. Ablikim et al. (BESIII Collaboration), Phys. Rev. Lett. 110, 252001 (2013).

[14] Z. Q. Liu et al. (Belle Collaboration), Phys. Rev. Lett. 110, 252002 (2013).

[15] D. Y. Chen, X. Liu, and T. Matsuki, Eur. Phys. J. C 72, 2008 (2012).

[16] M. Ablikim et al. (BESIII), Phys. Rev. D83, 012003 (2011).

[17] M. Ablikim et al. (BESIII), arXiv:1709.04627.

[18] B. Borasoy and R. Nissler, Eur. Phys. J. A26, 383 (2005).

[19] R. Escribano, P. Masjuan and J. J. Sanz-Cillero, JHEP 05, 094 (2011).

[20] P. Adlarson et al., arXiv:1708.04230.

[21] B. Kubis and S. P. Schneider, Eur. Phys. J. C62, 511 (2009).

[22] M. Ablikim et al. (BESIII), Phys. Rev. Lett. 118, 012001 (2017).

[23] D. J. Gross, S. B. Treiman and F. Wilczek, Phys. Rev. D19, 2188 (1979).

[24] B. Borasoy, U.-G. Meissner and R. Nissler, Phys. Lett. B643, 41 (2006).

[25] D. Alde et al. (Serpukhov-Brussels-Los Alamos-Annecy(LAPP)), Z. Phys. C36, 603 (1987).

[26] M. Ablikim et al. (BESIII), Phys. Rev. D92, 012001 (2015).

[27] L. G. Landsberg, Phys. Rept. 128, 301 (1985).

[28] M. Ablikim et al. (BESIII), Phys. Rev. Lett. 120, 242003 (2018).

[29] M. Gell-Mann, D. Sharp and W. G. Wagner, Phys. Rev. Lett. 8, 261 (1962).

[30] G. J. Gounaris and J. J. Sakurai, Phys. Rev. Lett. 21, 244 (1968).

[31] F. Stollenwerk, C. Hanhart, A. Kupsc, U. G. Meissner and A. Wirzba, Phys. Lett. B707, 184 (2012).

[32] P. Adlarson et al. (WASA-at-COSY), Phys. Lett. B707, 243 (2012).

[33] D. Babusci et al. (KLOE), Phys. Lett. B718, 910 (2013).

[34] B. Kubis and J. Plenter, Eur. Phys. J. C75, 283 (2015).

[35] M. Ablikim et al. [BESIII Collaboration], Phys. Rev. Lett. 121, 022001 (2018).

[36] N. N. Achasov, S. A. Devyanin, and G. N. Shestakov, Phys. Lett. 88B, 367 (1979).

[37] J. J. Wu and B. S. Zou, Phys. Rev. D 78, 074017 (2008).

[38] D. V. Bugg, V. V. Anisovich, A. V. Sarantsev, and B. S. Zou, Phys. Rev. D 50, 4412 (1994). 